\title{
Des effets des principaux jus de fruits à l'encontre des troubles sanguins, osseux, glandulaires, consécutifs à une insuffisance d'utilisation calcique
} par Yvonne DUPUIS, Pierre BRUN et Paul FOURNIER ${ }^{(1)}$

Société scientifique d'Hygiène alimentaire, Paris.

\begin{abstract}
Avec le développement du machinisme qui, tout à la fois, a facilité le transport rapide des denrées alimentaires et a sensiblement diminué le besoin en aliments énergétiques, la consommation des légumes et des fruits frais, a augmenté dans d'énormes proportions. Les nutritionistes qui ont observé avec attention cette évolution s'en sont attribué le principal mérite.

Tandis que de nouvelles conditions de vie remaniaient la condition alimentaire, la notion de besoins en prin. cipes nutritifs nouveaux se dégageait lentement des observations et des expériences. Ainsi, la question des besoins alimentaires, de moins en moins satisfaite à mesure qu'elle vieillissait en s'accrochant aux seuls dogmes des apports énergétique et azoté, s'était trou. vée requinquée par la notion d'autres besoins en minéraux, en oligo-minéraux et en vitamines. Et dans ce domaine de l'apport en éléments minéraux et vitaminiques, les fruits et 'légumes frais, les crudités, apparurent bientôt comme des denrées irremplaçables.
\end{abstract}

Est-ce à dire que nos connaissances en matière de nutrition sont achevées, qu'elles sont suffisamment profondes et complètes pour que, en toute sérénité, nous puissions mêler les principes nutritifs de telle sorte que le consommateur soit à l'abri d'une mauvaise surprise? L'histoire suivante, toute neuve, montre qu'il n'en est rien.

Divers troubles, certains très graves, sanguins, nerveux, osseux, endocriniens, apparaissent chez le jeune rat si le régime, bien composé, bien équilibré, ne contient pas de la vitamine D ou l'un quelconque des " composés de structure » dont le type est le lactose. Puisque de l'absence dans la ration de l'un de ces composés, découlent divers désordres fonctionnels, mais que la présence de l'un de ces corps suffit à maintenir l'état normal, c'est que l'animal ne peut se passer de ce composé, c'est-à-dire qu'il lui est indispensable $(\mathrm{I}, 2)$.

Par « composés de structure », nous désignons un ensemble de corps, le plus souvent glucidiques et naturels, mais qui peuvent être aussi des produits de synthèse sans existence naturelle, ou qui peuvent prendre naissance par dénaturation des glucides énergétique (3). Ces composés sont nombreux, divers, interchangeables au point que leur rôle semble indépendant de leur nature. Par leur activité sur l'absorption et 1'utilisation du calcium (4), les " glucides de structure " s'opposent aux glucides énergétiques dont l'incapacité du point de vue de l'utilisation calcique est remarquable.

Nous avons pensé qu'il convenait d'examiner les divers groupes d'aliments sous l'angle de l'utilisation calcique. Car de la teneur de la ration en " composés de structure " (ou en vitamine D), dépendent diverses fonctions essentielles telles que 1'ossification, la coagulation sanguine ou la conductibilité neuro-musculaire. Amorcé par l'étude de l'activité de produits laitiers sur l'ossification (5), 1'examen est présentement poursuivi au moyen des jus extraits des principaux fruits, raisin, pommes, cassis, orange.

\section{LES CONDITIONS EXPÉRIMENTALES}

I. Caractères des jus de fruits employés

Trois des quatre jus de fruits essayés au cours de cette expérience sont d'ori- gine industrielle. Ce sont les jus de pomme, de raisin et de cassis. Ces jus sont purs, naturels. Le quatrième, celui d'orange, a été préparé par nous-même par simple expression du fruit.
Le tableau I donne la composition sommaire de ces jus telle que l'analyse chimique nous la fournit.

Notons, au passage, la richesse du jus de raisin en glucides, la forte teneur

(I) Avec la collaboration technique de A. M. CROC. 
TABLEAU I

Composition sommaire de divers jus de fruits.

\begin{tabular}{|c|c|c|c|c|c|}
\hline \multirow{2}{*}{ Éléments dosés } & \multirow{2}{*}{$\begin{array}{c}\text { Mode } \\
\text { d'expression } \\
\text { p. 100 c } \mathrm{c}^{3}\end{array}$} & \multicolumn{4}{|c|}{ Jus de fruits analysés } \\
\hline & & Raisin & Pomme & Cassis & Orange \\
\hline 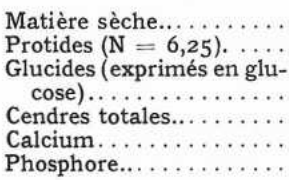 & $\begin{array}{l}\text { en } g \text {. } \\
\text { en } \mathrm{g} \text {. } \\
\text { en } g \text {. } \\
\text { en g. } \\
\text { en mg. } \\
\text { en } \mathrm{mg} \text {. }\end{array}$ & $\begin{array}{l}19,4 \\
0,46 \\
18,5 \\
0,3 \\
23 \\
14\end{array}$ & $\begin{array}{l}\text { I3, I } \\
0, \text { I6 } \\
\\
\text { II } 6 \\
0,2 \\
4 \\
5\end{array}$ & $\begin{array}{l}9,7 \\
0,23 \\
6 \\
0,5 \\
50 \\
6\end{array}$ & $\begin{array}{l}\text { 13 } \\
0,12 \\
\text { 10, } 5 \\
0,5 \\
\text { 10 } \\
\text { 16 }\end{array}$ \\
\hline
\end{tabular}

en calcium du jus de cassis, la pauvreté, par rapport aux autres, du jus de pomme, en phosphore et en calcium.

II. Les régimes d'expérience.

L'effet des divers jus est recherché par comparaison avec les performances physiologiques de deux régimes témoins :

$I^{\circ}$ Les Régimes "témoin 》:

a) le régime "amidon ». Ce régime, dont la composition détaillée figure dans le tableau 2, est un régime complet, équilibré. Sa teneur en calcium, de $580 \mathrm{mg}$ pour roo $\mathrm{g}$ de produit sec, est parfaitement normale. Ce calcium est offert, dans des conditions opti- males puisque le rapport des quantités de calcium et de phosphore qu'il contient est de $I, 6$.

Du fait de la purificarion très poussée de certains de ses constituants, notamment la caséine, ce régime est aussi dépourvu que possible de facteurs d'utilisation calcique, tels que la vitamine $D$ ou le lactose. Du fait de l'absence de ces facteurs, ce régime ne permet qu'une utilisation calcique très médiocre. C'est donc un régime témoin négatif.

b) Le régime "lactose ». Il s'agit, cette fois, d'un régime témoin positif, puisqu'il est revalorisé par la présence de lactose.

En fait, ce régime ne diffère du régime précédent, dit régime " amidon " que par la substitution de $20 \%$ de lac- tose à une proportion équivalente d'amidon.

$2^{\circ}$ Les régimes renfermant du jus de fruit.

Chacun des quatre jus de fruits essayés a été administré aux animaux à deux taux différents.

Pour la préparation des 2 régimes témoin, on mélange et on cuit ensemble I $\mathrm{kg}$ de régime sec et 2 litres d'eau. Au moyen de chacun des jus, on a préparé deux régimes différents, en tenant compte dans la proportion de régime sec employé, de la teneur du jus essayé en matière sèche.

- Un premier régime, dit $50 \%$, est préparé en ajoutant à une partie de matière sèche, une partie d'eau et une partie de jus de fruit. Ainsi, le jus de fruit ne constitue que la moitié du liquide employé pour la préparation du régime. De là le terme de régime $50 \%$. - Un second régime dit $100 \%$ est préparé en additionnant une partie du régime sec de deux parties de jus. Le jus de fruit constitue toute la partie liquide, soit $100 \%$ du liquide du régime.

III. Les animaux d'expérience.

L'expérience a porté sur 120 rats répartis de façon homogène en Io lots : 2 lots "témoin ", l'un " amidon ", l'autre "lactose »; 8 lots dont le ré-

TABLEAU 2

Composition des régimes (en grammes pour 300 grammes de régime humide).

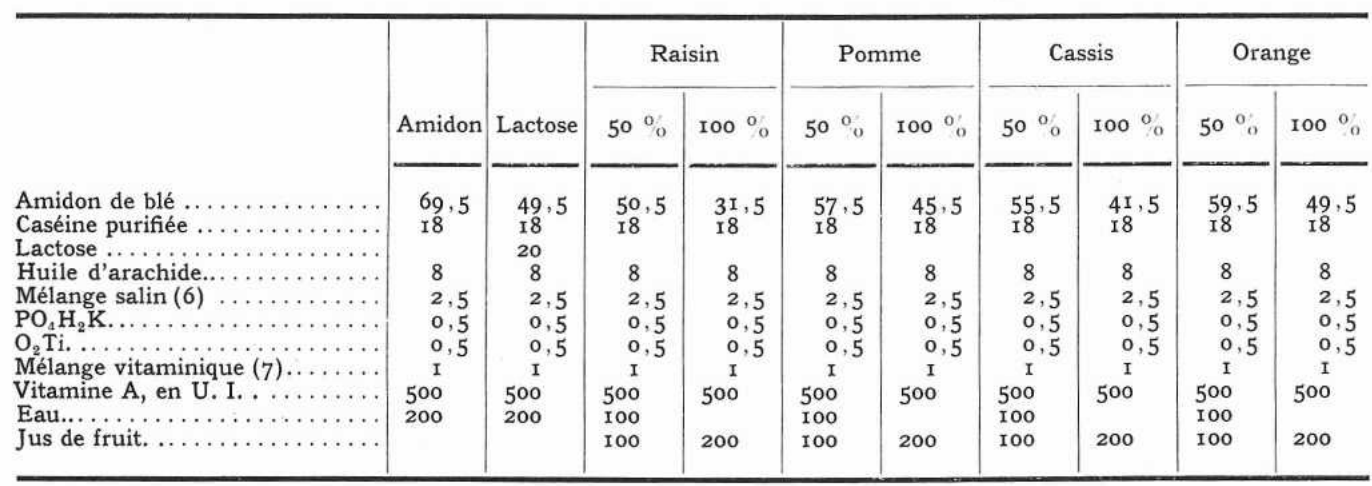




\section{Lu dans Fruits, il y a 50 ans ...}

gime contient le jus de fruit, à raison de 2 lots pour chaque jus.

Les animaux sont mis en lot dès le sevrage. L'expérience dure 6 semaines pendant lesquelles ils consomment, à volonté, une quantité connue de régime.

Les animaux sont soumis à divers tests dans les conditions opératoires suivantes :

$\mathrm{I}^{\circ}$ Les bilans calciques.

Pendant deux jours consécutifs de la première et de la troisième semaine d'expérience, 6 animaux de chaque lot sont mis individuellement dans un dispositif qui permet de récolter séparément les fèces et les urines. Le calcium est dosé dans ces excreta, ainsi que dans le régime. La présence d' $\mathrm{O}_{2} \mathrm{Tl}(8)$ dans le régime et les fèces permet de calculer directement le coefficient d'absorption intestinale du calcium au moyen de la formule : coefficient d'absorption =

$\frac{\mathrm{Ca} / \mathrm{O}_{2} \mathrm{Ti} \text { du régime }-\mathrm{Ca} / \mathrm{O}_{2} \mathrm{Ti} \text { des fèces }}{\mathrm{Ca} / \mathrm{O} \mathrm{Tidu} \text { régime }} \times 100$

Compte tenu du résultat de ces analyses et de la quantité de régime consommé, il est aisé de calculer le bilan calcique journalier.

$2^{\circ}$ Détermination de la calcémie.

Une méthode extrêmement sensible et précise (9) permet de déterminer la teneur du sérum en calcium à partir de quelques gouttes de sang prélevées sous anesthésie par incision de la queue. Trois déterminations ont été faites, à la fin des $2^{\mathrm{e}}, 4^{\mathrm{e}}$ et $6^{\mathrm{e}}$ semaines d'expérience.

\section{$3^{\circ}$ Examen du tibia.}

A la fin des $3^{\mathrm{e}}$ et $6^{\mathrm{e}}$ semaines d'expérience, 6 animaux de chaque lot son sacrifiés. Les tibias sont prélevés, nettoyés, fendus longitudinalement. L'aspect des régions métaphysaires et épiphysaires est noté afin d'évaluer le degré de rachitisme osseux.

\section{$4^{\circ}$ Examen des parathyrö̈des.}

L'animal sacrifié, on dissèque la face antérieure du cou. On extirpe la tra chée et, avec elle, les glandes thyroïde et parathyroïdes. Ces dernières fon l'objet d'un examen minutieux afin d'en apprécier les dimensions.

\section{LES RÉSULTATS}

Les résultats sont présentés dans les tableaux 3 et 4 , l'un qui fait état des divers éléments du bilan calcique, l'autre qui groupe les valeurs de la calcémie ainsi que les observations relatives au degré de rachitisme osseux et aux dimensions des parathyroïdes.

Dans l'ensemble, ces résultats permettent d'affirmer une certaine influence favorable des jus de fruits sur l'utilisation du calcium, Cependant, l'efficacité des divers jus est plus ou moins manifeste selon le test auquel on se réfère.

\section{Io Les bilans calciques,}

Pour chaque lot, nous avons fait état des quantités journalières de calcium ingéré, absorbé et urinaire. La différence entre le calcium absorbé et le calcium urinaire fournit le calcium retenu. Le coefficient d'absorption est calculé d'après la formule :

$$
\frac{\text { Ca absorbé }}{\text { Ca ingéré }} \times 100
$$

Le coefficient d'utilisation est le rapport :

$$
\text { Ca retenu } \times \text { roo }
$$

Après une semaine d'expérience, certains lots n'absorbent et ne retiennent pas davantage de calcium que le lot amidon. Ainsi se comportent les rats qui reçoivent les régimes : raisin $50 \%$, cassis $50 \%$ et $100 \%$ et orange $50 \%$ et $100 \%$. Par contre, on note un effet important sur l'utilisation calcique du jus de raisin incorporé à $100 \%$ dans le régime, ainsi que du jus de pomme incorporé à raison de $50 \%$. Cet effet favorable du jus de pomme sur l'absorption et la rétention du calcium est confirmé par l'activité remarquable du régime renfermant ce jus à I0o \% 。

Dans l'ensemble, après 3 semaines d'expérience. les jus révèlent tous une certaine activité sur l'utilisation calcique. Comparés au régime témoin seuls, pour les dosages les plus faible auxquels nous les employons à $50 \%$ les jus de raisin et de cassis n'améliorent pas les bilans. Dans les 6 autres cas, c'est-à-dire, pour les jus de pomme et d'orange, aux deux taux de $50 \%$ et de $100 \%$, où nous les essayons, et pour les jus de raisin et de cassis au maximum de leur emploi (roo \%), l'effet sur l'utilisation calcique est toujours nette, parfois considérable comme c'est encore le cas pour le jus de pomme utilisé en proportion de $100 \%$.

$2^{\circ}$ Résultats des calcémies.

Les variations du taux du calcium sanguin, indiquent bien, chez les rats qui reçoivent le régime amidon, une chute profonde et durable de la calcémie, antérieurement signalée (10). La présence de $20 \%$ de lactose dans le régime s'oppose complètement à cette chute, c'est-à-dire que, chez tous les rats dont le régime comporte du lactose, le calcium sanguin demeure au niveau de $100 \mathrm{mg} /$ litre. Rappelons que ce niveau est une des principales constantes physiologiques non seulement pour le rat ou l'homme, mais pour toutes les espèces de mammifères. Rappelons aussi que la baisse de la calcémie se fait aux dépens du calcium ionisé et que, de ce fait, entraîne des perturbations du fonctionnement neuro-musculaire.

Dans quelle mesure la présence de jus de fruit dans le régime va-t-elle s'opposer à la diminution de la calcémie ? La première partie du tableau 4 permet de répondre à la question. En fait la réponse n'est pas absolument 
nette. Après deux semaines pendant lesquelles les rats des différents lots ont reçu le régime amidon seul, ou le régime renfermant soit du lactose, soit un jus de fruit, seuls ceux qui reçoivent du lactose maintiennent la position normale. Tous les autres ont amorcé un repli qui, notamment pour les lots au jus d'orange, est important. Puis, chez tous les lots, sauf le lot lactose, la calcémie continue de s'abaisser. On note cependant très nettement que la pré-

TABLEAU 3

Les bilans calciques.

\begin{tabular}{|c|c|c|c|c|c|c|}
\hline \multirow{2}{*}{ Lots } & \multicolumn{4}{|c|}{ Quantité de calcium (mg/j) } & \multicolumn{2}{|c|}{ Coefficient (en \%) } \\
\hline & Ingéré & Absorbé & Urinaire & Retenu & absorption & Utilisation \\
\hline re semaine. & & & & & & \\
\hline 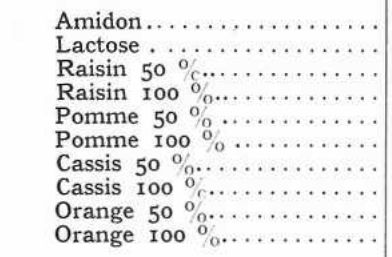 & $\begin{array}{l}50 \\
48 \\
54 \\
49 \\
58 \\
54 \\
55 \\
49 \\
48 \\
40\end{array}$ & $\begin{array}{l}25 \\
39 \\
26 \\
29 \\
33 \\
36 \\
26 \\
22 \\
23 \\
24\end{array}$ & $\begin{array}{l}2 \\
I \\
I \\
I \\
2 \\
2 \\
2 \\
I \\
I \\
I\end{array}$ & $\begin{array}{l}23 \\
38 \\
25 \\
28 \\
31 \\
34 \\
24 \\
21 \\
22 \\
23\end{array}$ & $\begin{array}{l}50 \\
81 \\
48 \\
59 \\
57 \\
67 \\
47 \\
45 \\
48 \\
60\end{array}$ & $\begin{array}{l}46 \\
79 \\
46 \\
57 \\
53 \\
63 \\
44 \\
44 \\
46 \\
57\end{array}$ \\
\hline $3^{\mathrm{e}}$ semaine. & & & & & & \\
\hline 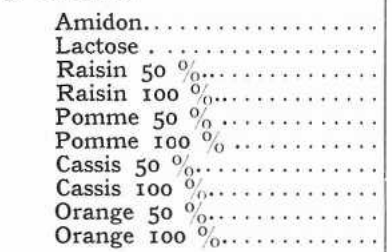 & $\begin{array}{l}51 \\
57 \\
59 \\
54 \\
63 \\
58 \\
62 \\
63 \\
52 \\
53\end{array}$ & $\begin{array}{l}16 \\
35 \\
18 \\
23 \\
24 \\
29 \\
19 \\
25 \\
23 \\
23\end{array}$ & $\begin{array}{l}\text { I } \\
2 \\
\text { I } \\
I \\
I \\
2 \\
2 \\
2 \\
I \\
I\end{array}$ & $\begin{array}{l}15 \\
33 \\
17 \\
22 \\
23 \\
27 \\
17 \\
23 \\
22 \\
22\end{array}$ & $\begin{array}{l}3 I \\
6 I \\
3 I \\
43 \\
38 \\
50 \\
3 I \\
40 \\
44 \\
43\end{array}$ & $\begin{array}{l}29 \\
58 \\
29 \\
41 \\
36 \\
47 \\
27 \\
37 \\
42 \\
41\end{array}$ \\
\hline
\end{tabular}

TABLEAU 4

Résultats de la calcémie et de l'observation des tibias et des glandes parathyrö̈des.

\begin{tabular}{|c|c|c|c|c|c|c|c|}
\hline Tests & \multicolumn{3}{|c|}{ Calcémie (mg/1) } & \multicolumn{2}{|c|}{ Degré de rachitisme } & \multicolumn{2}{|c|}{ Caractères des parathyroïdes } \\
\hline $\begin{array}{c}\text { Semaines } \\
\text { d'expériences }\end{array}$ & 2 & 4 & 6 & 3 & 6 & 3 & 6 \\
\hline Lots & & & & & & & \\
\hline 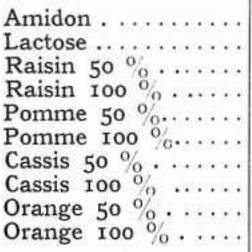 & $\begin{array}{l}86 \\
98 \\
82 \\
91 \\
82 \\
89 \\
86 \\
81 \\
79 \\
78\end{array}$ & $\begin{array}{l}68 \\
97 \\
75 \\
80 \\
78 \\
87 \\
74 \\
75 \\
75 \\
76\end{array}$ & $\begin{array}{r}66 \\
\text { 102 } \\
87 \\
84 \\
83 \\
84 \\
86 \\
88 \\
89 \\
93\end{array}$ & $\begin{array}{c}+++t+ \\
0 \\
++ \\
++ \\
++ \\
0 \\
+++ \\
++ \\
+++ \\
+++\end{array}$ & $\begin{array}{c}++ \\
0 \\
+ \\
0 \\
+ \\
0 \\
+ \\
+ \\
++ \\
+\end{array}$ & $\begin{array}{l}\text { diverses } \\
\text { invisibles } \\
\text { diverses } \\
\text { visibles } \\
\text { visibles } \\
\text { visibles } \\
\text { grosses } \\
\text { diverses } \\
\text { diverses } \\
\text { diverses }\end{array}$ & $\begin{array}{l}\text { hypertrophié } \\
\text { invisibles } \\
\text { diverses } \\
\text { invisibles } \\
\text { visibles } \\
\text { invisibles } \\
\text { visibles } \\
\text { visibles } \\
\text { diverses } \\
\text { visibles }\end{array}$ \\
\hline
\end{tabular}




\section{Lu dans Fruits, il y a 50 ans ...}

sence du jus de fruit dans le régime tend à freiner parfois efficacement, dans d'autres cas modérément, cette diminution du calcium sanguin.

Mais c'est à la fin de l'expérience, soit après 6 semaines, que les jus de fruits manifestent le plus clairement leur action sur le calcium sanguin. Sans prétendre à 1'efficacité remarquable du lactose, pris comme référence, on voit que, pour tous les lots auxquels correspond un régime contenant un jus de fruit, la calcémie est à un niveau plus élevé que le bas niveau des animaux du lot témoin. Dans la plupart des cas, on observe même une remontée plus ou moins notable de la calcémie.

$3^{\circ}$ Évaluation du degré de rachitisme.

En dépit du calcium qui leur est offert dans des conditions optimales, les rats du lot témoin présentent tous des signes d'un rachitisme osseux très prononcé.

Le signe le plus grave, le plus apparent aussi, lorsque l'on examine une coupe longitudinale du tibia, c'est le développement très exagéré et très irrégulier du cartilage épiphysaire attenant au plateau tibial. Cette zone cartilagineuse est épaisse, si considérablement en certains points qu'elle forme des renfements et confère à la ligne d'ossification un tracé anormalement sinueux. Sous ce cartilage anormal, les travées osseuses sont épaisses, tordues, contrastant avec la régularité et le parallélisme qui caractérisent la disposition des travées dans l'ossification normale. Très souvent, on note aussi une décoloration de la zone métaphysaire.
Ce sont ces anomalies, caractéristiques de l'os rachitique, anomalies très prononcées après 3 semaines d'expérience que nous avons notées de ++++++ , indiquant ainsi que les signes positifs de rachitisme sont nombreux.

Avec le temps, les signes de rachitisme tendent à s'estomper ce qui se traduit, après 6 semaines d'expérience, par une diminution des signes positifs $(+++)$.

La présence de lactose dans le régime des animaux protège complètement l'animal de toute atteinte de rachitisme osseux. Ce qui, sur le tableau, se traduit par le signe 0 .

Que se passe-t-il lorsque le régime des animaux renferme un jus de fruit ? Dans tous les cas et sans la moindre équivoque, on peut affirmer que l'état des os est bien meilleur que celui des animaux témoin amidon. Cependant, pour nette qu'elle soit, la protection osseuse exercée par les jus de fruits n'atteint que très rarement à 1'efficacité totale du lactose.

Après 3 semaines de régime, la plupart des animaux dont le régime comporte un jus de fruit, présente des signes de rachitisme. Mais ce rachitisme est beaucoup plus discret que celui des animaux au régime amidon. Aux vastes poches qui déformaient par leur ampleur le cartilage épiphysaire, ont fait place des élargissements, anormaux certes, mais assez discrets. Et dans le cas du jus de pomme employé à $100 \%$, il n'existe aucun signe de rachitisme, les os présentant tous les caractères du développement normal.

Après 6 semaines d'expérience, une proportion notable des animaux dont le régime comporte un jus de fruit n'a aucun signe de rachitisme osseux. D'autres au contraire, dans le même lot, ne présentent pas des tibias absolument normaux. D'où cette notation d'un léger état rachitique qui se traduit en général, dans le tableau, par la présence d'un seul + qui traduit un aspect du tibia beaucoup plus favorable que pour les rats du régime amidon $(+++)$.

$4^{\circ}$ Caractères des glandes parathyroïdes.

Une carence matérielle en calcium (par privation) ou une carence fonctionnelle en calcium (par défaut d'utilisation), se traduisent par une hypertrophie des glandes parathyroïdes (I). Ceci est surtout manifeste après 6 semaines d'expériences.

En fait, sauf dans le cas d'une hypertrophie importante, il est assez difficile d'évaluer les dimensions de ces glandes. Elles sont si petites, incluses, qu'elles sont difficiles à apercevoir, englobées dans la masse, elle-même modeste, de la glande thyroide. Ainsi chez les animaux dont le régime comporte du lactose, les parathyroïdes sont pratiquemnet invisibles, à l'examen direct.

De cette expérience, il ressort que les glandes parathyroïdes des animaux dont le régime comporte des jus de fruits, sont en général, intermédiaires entre la taille surdéveloppée des rats $\mathrm{du}$ lot amidon, toujours bien visibles et faisant nettement saillie sur le contour arrondi de la thyroïde et les dimensions des glandes parathyroïdes des rats du lot lactose, si réduites qu'elles ne sont pratiquement pas visibles à l'œil nu.

\section{INTERPRETATION ET DISCUSSION}

\section{Comparaison des divers tests employés.}

Tout d'abord il est hors de doute que les jus de fruits possèdent une activité certaine sur l'utilisation calcique. $\mathrm{Ce}$ fait ressort clairement dans tous les compartiments de cette expérimentation.
I) Les jus de fruits améliorent l'absorption et la rétention de calcium.

2) Les jus de fruits freinent la baisse du taux du calcium sanguin consécutive à l'absence, dans le régime, de facteurs d'utilisation calcique.

3) Les jus de fruits ont une influence manifeste sur le déroulement normal des processus de l'ossification.
4) Les jus de fruits tendent à maintenir l'aspect habituel et le fonctionnement normal des glandes parathyroïdes.

Mais selon le test employé pour juger de l'efficacité des jus de fruits à l'égard de l'utilisation du calcium, on note des différences importantes.

Sur la partie directement matérielle de l'utilisation calcique, c'est-à-dire sur 


\section{Lu dans Fruits, il y a 50 ans ...}

celle que l'on juge d'après ce que retient l'animal, l'efficacité du jus de fruit est assez modeste. Certes, les rats dont le régime comporte du jus de raisin, de cassis, d'orange et surtout du jus de pomme, voient, au moins en certains temps de 1'expérience, leur rétention calcique augmenter. Mais assez souvent, par rapport aux rats du lot amidon, l'accroissement de la rétention calcique est discrète.

L'influence des jus de fruits sur le maintien de la constante calcique du sérum, est indéniable. Elle n'est cependant que partielle et, dans un certain sens, différée, car ce n'est qu'à la fin de l'expérience que l'écart entre les témoins amidon et les rats qui reçoivent les jus de fruits, est notable. Néanmoins, en aucun cas, les jus de fruits ne permettent à la calcémie de demeurer ou de retrouver le niveau physiologiquement constant.

Ainsi, à juger seulement d'après les échanges matériels du calcium, c'està-dire d'après les entrées et les sorties de calcium ou d'après le calcium sanguin, l'efficacité des jus de fruits sur les fonctions calciques serait légère.

Mais il est d'autres critères d'après lesquels il est possible de juger le fonctionnement calcique de l'animal. C'est par exemple l'état du squelette ou de certaines glandes étroitement impliquées dans l'utilisation calcique. Et dans ces deux cas - l'état du squelette et l'état des parathyroïdes - l'activité des jus de fruits apparaît fortement.

Avant de rechercher les raisons ou la signification de ces différences dans le degré d'action des jus de fruits à l'égard des divers critères de 1'utilisation calcique, notons toutefois que, en général, pour un jus de fruit donné, c'est la forte dose qui manifeste le plus d'activité. Notons aussi que c'est le jus qui est le plus actif à l'égard de l'un des critères qui l'est aussi à l'égard des autres tests. C'est dans la présente expérience le cas du jus de pomme.

II. Rapports et divergences entre l'absorption et l'utilisation du calcium.

En bref, il ressort de cette expérience que l'influence des jus de fruits sur l'uti- lisation calcique, notamment sur l'ossification, est beaucoup plus nette que leur influence sur l'absorption et la rétention calcique.

C'est une suite ininterrompue de dix ans de travaux qui nous ont conduits à montrer pratiquement que :

I ${ }^{\circ}$ Un grand nombre de substances rassemblées dans un groupe de « composé de structure " améliorent l'absorption et la rétention du calcium (3)

$2^{\circ}$ Un régime dépourvu de vitamine D ou de "composé de structure » permet à l'animal d'absorber et de retenir une quantité importante de calcium. Néanmoins, l'animal ne maintient pa sa calcémie au niveau normal et il présente des signes de rachitisme osseux (4)

$3^{\circ}$ Des troubles sanguins, nerveux, osseux, glandulaires, troubles liés à une insuffisance de l'utilisation du calcium, apparaissent chez le jeune rat, si le régime bien composé, de teneur optimale en calcium, est dépourvu de vitamine D ou de l'un des " composés de structure " dont le type est le lactose. Ces composés doivent donc être considérés comme indispensables $(I, 2)$.

Demandons-nous, à travers cette progression de nos connaissances relatives à 1'utilisation calcique, quelle peut être la signification des résultats de cette expérience.

Un premier point émerge de cet ensemble de travaux : c'est l'incapacité du calcium du régime d'assurer les fonctions calciques essentielles. L'apport calcique exogène n'est donc pas, comme on l'a cru longtemps, le point essentiel de 1'utilisation calcique (I0).

Un second fait, plein de promesse, se dégage de nos travaux actuels. Les animaux du lot amidon absorbent et retiennent d'importantes quantités de calcium (4). Cependant leur calcémie s'effondre et leur squelette est ravagé de rachitisme. D'où ce second point : il ne suffirait pas certes d'offrir du calcium à l'animal, il ne suffirait pas non plus que ce calcium soit absorbé et retenu pour que les fonctions calciques soient satisfaites. L'essentiel de 1'utilisation calcique ne reposerait pas sur l'apport ou la rétention du calcium mais cette utilisation reposerait essentiellement sur les facteurs d'utilisation.

Or, ces facteurs, ce sont, en plus de la vitamine $\mathrm{D}$, tous les composés de structure dont le type est le lactose. Naturels ou non, préformés ou néoformés par dénaturation des glucides énergétiques, ces composés de structure sont nombreux, divers, interchangeables, au point que leur fonction semble ne pas dépendre de leur nature (I). Ils sont bien les facteurs exogènes indispensables de l'utilisation du calcium.

III. Les raisons de l'activité des jus de fruits sur l'utilisation du calcium.

Admettre sans réserve la primauté des facteurs d'utilisation dans la question essentielle du métabolisme calcique, ne nous dit pas pourquoi les jus de fruits révèlent surtout leur activité à l'égard de l'ossification.

Et d'abord, demandons-nous si les jus de raisin, de pomme, de cassis et d'orange renferment ces facteurs d'utilisation calcique.

Il n'apparaît pas, à la vue d'analyses, il est vrai, sommaires, que les jus de fruits puissent renfermer d'importantes quantités de ces composés de structure, principes jugés sans intérêt avant d'être identifiés comme facteurs indispensables de l'utilisation du calcium. Sans doute, l'analyse des jus de fruits n'at-elle jamais été dirigée vers la recherche et le dosage des divers corps capables de tenir le rôle de " composés de structure ». D'autant que la détection de certains d'entre eux est un problème d'analyse délicat. Sans doute aussi, 1'activité somme toute modeste des jus de fruits à l'égard de l'utilisation calcique s'accommoderait-elle d'une teneur assez faible de ces jus " composés de structure ". Seul un travail analytique long et difficile permettrait de faire le point de cette importante question.

Mais sans vouloir pousser à fond les diverses hypothèses que suggèrent les présents résultats, qu'il nous soit permis de formuler deux propos:

$I^{0} \mathrm{~A}$ considérer les différences d'activité du jus de fruit à l'égard des divers critères de 1'utilisation calcique, à considérer surtout leur influence favorable sur l'ossification, on peut se demander si certains facteurs actifs ne sont pas surtout efficaces a légard de certain 


\section{Lu dans Fruits, il y a 50 ans ...}

tests. Ainsi, comprendrait-on aisément qu'une meilleure absorption ne commanderait pas automatiquement une meilleure ossification.

$2^{\circ}$ Tous ces jus de fruits renferment des acides organiques qui, pour diffé- rents de nature qu'ils soient, n'en demeurent pas moins physiologiquement apparentés. Au moins certains d'entre eux comme les acides malique et citrique. Quelle est l'action de ces composés sur l'utilisation du calcium ?
Certes la question que nous avons abordée antérieurement (II) a donné lieu à des travaux et des réponses trop disparates pour qu'elle ne soit pas reprise entièrement sous l'angle des connaissances nouvelles précédemment énoncées.

\section{CONCLUSION}

Une étude biologique approfondie a été consacrée à l'action de divers jus de fruits sur l'utilisation du calcium.

Les essais ont porté sur les jus de raisin, de pomme, de cassis et d'orange. Leur efficacité sur l'utilisation calcique a été jugée :

$\mathrm{I}^{0}$ d'après la valeur des bilans du calcium ;

$2^{\circ}$ d'après le niveau de la calcémie ; $3^{\circ}$ d'après l'aspect des os longs ; $4^{\circ}$ d'après la taille des glandes parathyroïdes.

Des troubles graves : osseux, sanguins, glandulaires, consécutifs à une mauvaise utilisation calcique apparaissent si le régime ne renferme pas de facteurs d'utilisation calcique. Dans quelle mesure les jus de fruits peuventils prévenir l'apparition de ces troubles?

Les jus de fruits accroissent d'une façon modérée l'absorption et la rétention du calcium; ils s'opposent dans une certaine mesure à la chute de la calcémie et à l'hypertrophie des glandes parathyroïdes. Mais c'est à l'égard de l'ossification qu'ils se révèlent le plus actifs.

Il n'est pas possible de dire pour l'instant si l'action des jus de fruits sur l'utilisation calcique, action d'ailleurs modérée, doit être rapportée à des composés glucidiques appartenant au groupe des composés de structure ou à la présence de divers acides organiques.
(I) Fournier (P.), Dupuis (Y.). - Facteurs exogènes indispensables de l'utilisation du calcium. C. $R$. Acad. Sc., I96a, sables de l'utilisatio
254 , séance du 7 mai.

(2) Dupuis (Y.), Fournier (P.). - Lactose and the absorption of calcium and strontium. Conférence faite le ${ }_{5} 5$ mai 1962 par Y. Dupuis, à Ithaca (New York) sur le " transport du par Y. Dupuis, à Ithaca (New York) sur le " transport dy
calcium et du strontium à travers les membranes biologiques A paraitre en 1962 dans * Annals of the New York Academy of Sciences

(3) Fournier (P.), Dupuis (Y.). - Divers composés glucidiques possèdent-ils des qualités plastiques particulières? Bull. Soc. Sc. Hyg. alim., 1960, 48, 33 .

(4) Dupuis (Y.), Brun (P.), Fournier (P.). - Étude de l'activité du lactose, facteur exogène de l'utilisation du calcium, en fonction de la dose administrée. C. R. Acad. Sc., 1962, 254 2230 .

(5) Dupuis (Y.), Brun (P.), Fournier (P.). - Yoghourt et utilisation du calcium. Bull. Soc. Sc. Hyg. alim., 1962, 50, 55 .
(6) Hubbel (R.), Mexpel (L.), Wakemax (A.) - A new salt mixture for use in experimental diets. $J$. Nutr., 1937, 14, 273

(7) Fischer (J.). - Effects of feeding diets containing lactose, agar, cellulose, raw potato starch or arabinose on the dry weights of cleaned gastrointestinal tract organes in the Rat. Amer. J. Physiol., 1957, 188, 550.

(8) Fournier (P.). - De l'emploi de l'oxyde de titane pour l'étude quantitative de l'absorption intestinale. C. R. Acad. Sc., I950, 231, I343.

(9) KingsLey (G.) et RobNett (O.). - New dye method for direct photometric determination of calcium. J. Clin. Path., 1957, 27, 223 .

(10) Dupurs (Y.). - Perturbations biochimiques observées chez le jeune Rat soumis à un régime privé de facteurs d'utilisation du calcium. Action préventive de la vitamine D et du lactose. C. R. Acad. Sc., 1962, 254, séance du 30 avril.

(II) BRUN (P.). - Influence des jus de raisin sur la rétention du calcium chez le jeune Rat. Colloque sur le jus de raisin en biologie et en thérapeutique. Montpellier, 1961, édit. Gauthier-Villars, Paris, 1962. 